\title{
A Call Admission Control for Service Differentiation and Fairness Management in WDM Grooming Networks*
}

\author{
Kayvan Mosharaf, Jérôme Talim and Ioannis Lambadaris \\ Depart. of Systems and Computer Engineering, \\ Carleton University, Ottawa, ON, Canada \\ \{mosharaf,jtalim,ioannis\}@ sce.carleton.ca
}

\begin{abstract}
We investigate a Call Admission Control (CAC) mechanism to provide fairness control and service differentiation in a WDM network with grooming capabilities. A WDM grooming network can handle different classes of traffic streams which differ by their bandwidth requirements. We assume that for each class, call interarrival and holding times are exponentially distributed. Using a Markov Decision Process approach, an optimal CAC policy is derived to provide fairness in the network. The Policy Iteration algorithm is used to numerically compute the optimal policy. Furthermore, we propose a heuristic decomposition algorithm with lower computational complexity and very good performance. Simulation results compare the performance of our proposed policy, with that of Complete Sharing and Complete Partitioning policies. Comparisons show that our proposed policy provide the best performance in most cases. Although this approach is motivated by WDM networks, it may be deployed to determine the optimal resource allocation in many problems in wireless and wired telecommunications systems.
\end{abstract}

\section{Introduction}

Wavelength Division Multiplexing (WDM) with grooming capabilities is a promising candidate to handle the bandwidth demand of future Wide Area Networks. In WDM networks, each optical path must be established with a specific wavelength between each source-destination pair. This is known as wavelength continuity constraint and can be relaxed by using wavelength converters at intermediate nodes. Although each wavelength can have a transmission capacity up to ten gigabit per second (e.g., OC-48 or OC-192), some traffic streams may require lower bandwidth (e.g., OC-3 or

${ }^{*}$ This work was supported by a grant from MITACS (Mathematics for Information Technology and Complex Systems)
OC-12). To utilize the wavelengths more efficiently, traffic grooming is deployed to share the capacity of a wavelength among users with different bandwidth granularities by multiplexing/demultiplexing the lower rate traffic onto high capacity wavelengths. Therefore, the bandwidth of each wavelength may be divided into a set of time slots. Depending on the bandwidth requirement of a traffic demand, one or multiple time slots can be used to accommodate the traffic [13].

In WDM grooming networks, nodes are equipped by Optical Add/Drop Multiplexers (OADMs) and Optical Crossconnects (OXCs). OXCs can have wavelength converter (WC) and time-slot interchanger (TSI) devices. TSIs are used to switch a set of time slots from one wavelength to another, whereas WCs convert an incoming wavelength to another outgoing wavelength. In this study, we assume that OXCs are not provided with these devices, because using TSIs and WCs are not cost-effective yet.

Many heuristic algorithms such as First-Fit, Most-Used, Max-Sum and Random wavelength assignment have already been proposed for the case when each call occupies the whole wavelength [12]. The objective of these algorithms is typically to minimize the overall call blocking probabilities in a single-rate WDM network. Some complementary studies related to this problem can be found in $[1,3,14]$. A few analytical models have been proposed in the literature to compute the blocking probabilities in multirate WDM networks with grooming capabilities [8-10]. In [10], a capacity correlation model is presented for evaluation of blocking probabilities in a multi-hop single wavelength path, whereas [9] discusses the numerical computation of the blocking rates in a two-class network without capacity correlation between the links. An analytical model is provided in [8] to evaluate the blocking performance in WDM-Time Division Multiplexing (TDM) networks. The fairness issue in WDM grooming networks is investigated in [11]. In this study, a CAC mechanism is used to improve the fairness in the network. The CAC algorithm implemented based on current statistics results (i.e., 
blocking probabilities of different classes in the whole network). This mechanism can be used only after the network passes the transient condition.

The purpose of this paper is to develop a call admission control to provide fairness control and service differentiation in WDM grooming networks. We first define a Markov Decision Process (MDP), with the objective of maximizing class-based utilization in a single-wavelength singlelink network. Based on MDP formulation, a CAC scheme can be determined as a function of current capacity usage on each wavelength. The Policy Iteration algorithm can be deployed to determine the optimal CAC policy [6]. Since implementing an MDP-based CAC mechanism is very difficult for a complex network topology, we develop approximations and make some assumptions to extend the result of the single-wavelength single-link network for applying to multi-wavelength multi-link tandem and ring networks. Thus, we concentrate on developing an estimation of CAC scheme which provides accurate results with lower computational complexity. In many admission control and resource allocation problems, it has been shown that under some conditions, the optimal policy of an MDP exists and it is stationary and monotone [5].

The rest of the paper is organized as follows. In Section II, we introduce the network model. Section III deals with MDP formulation for a single-wavelength single-link network and the discounted reward function associated with the problem in the infinite horizon case. Section IV shows the structure of optimal policy and Section V describes our proposed heuristic algorithm. Section VI compares the performance of the proposed policy with other standard policies. Conclusions are presented in Section VII.

\section{Network model}

We consider a WDM grooming network consisting of $L$ links. The network includes $M$ origin-destination $(o-d)$ pairs, indexed by $m=1,2, . ., M$. All nodes are equipped by OXCs and OADMs without TSI and WC devices. Each link carries one fiber with $W$ wavelengths. And each wavelength includes $T$ time slots. The network supports $K$ classes of traffic streams, $c_{1}, c_{2}, \ldots, c_{K}$, which differ by their bandwidth requirements. Class $c_{k}$ traffic requires $t_{k}$ time slots to be established. We also use the following assumptions.

- For each o-d pair, class $c_{k}$ arrivals are distributed according to a Poisson process with rate $\lambda_{k}$.

- The call holding time of class $c_{k}$ is exponentially distributed with mean $\mu_{k}^{-1}$. Unless otherwise stated, we assume $\mu_{k}^{-1}=1$.

- Any arriving call from any class is blocked when no wavelength has $t_{k}$ available time slots.
- Blocked calls do not interfere with the system.

- The switching nodes are non-blocking

Since calls from different classes compete for access to the network, fairness control and resource sharing management are very challenging issues. Complete Sharing (CS) and Complete Partitioning (CP) policies are two of the wellknown capacity access control schemes [7].

CS policy does not reserve resources such as bandwidth or wavelength to any class of calls. In addition, an arriving call is accepted if at least one wavelength has enough free slots to carry that call. In this case, calls with lower bandwidth requirements (respectively, higher arrival rates) may starve calls with higher bandwidth requirements (respectively, lower arrival rates). Therefore, the system is unfair in the sense that there is a noticeable difference between the blocking probabilities experienced by different classes of users. Here, the objective of fairness is to maintain this difference as small as possible, so that all classes experience the same blocking probability.

On the other hand, when CP policy is implemented, each class is dedicated a portion of the resources that cannot be used by calls from other classes. Hence, it provides fairness and supports service differentiation. However, CP policy may not maximize the overall utilization of the available resources. Our goal, then, is to design a connection admission policy which provides fairness and maximizes the overall utilization.

In the following sections, we formulate this problem in an MDP framework. Since the MDP-based formulation for a large network is computationally infeasible, we first formulate the problem for a single-wavelength single-link network to derive the optimal policy. By using the properties of the optimal policy and developing approximations, we propose a quite simple heuristic algorithm to support fairness issue in the network.

\section{Problem formulation for a single-link single- wavelength network}

In this section, we consider a single-link path which has only one wavelength to carry traffic streams. Let $n_{k}$ denote the number of class $c_{k}$ calls currently in the system. Therefore, a $k$-component vector, $\left(n_{1}, n_{2}, \ldots, n_{k}\right)$, can completely characterize the system. Let

$$
S=\left\{\left(n_{1}, n_{2}, . ., n_{k}\right) \mid \sum_{k=1}^{K} t_{k} n_{k} \leq T\right\}
$$

be the system state space and $s_{t}$ denote the state of the system at time $t$. Based on the statistical assumptions, $\left\{s_{t}, t \geq 0\right\}$ is a continuous-time Markov chain whose transitions are either the event of an arrival or a departure of 
a call. For the sake of simplicity, the index $t$ is no longer mentioned in the notation.

In order to simplify the notation, the following operators are introduced:

- $A_{k}: S \mapsto S, k=1,2, \ldots, K$ : Arrival operator, describing the change of the state of the system at the arrival time of a $c_{k}$ user.

$$
\text { - } A_{k} s=\left(n_{1}, n_{2}, . ., n_{k}+1, \ldots, n_{K}\right) \text {. }
$$

- $D_{k}: S \mapsto S, k=1,2, \ldots, K$ : Departure operator, describing the change of the state of the system at the departure time of a $c_{k}$ user.

$$
\text { - } D_{k} s=\left(n_{1}, n_{2}, . ., n_{k}-1, \ldots, n_{K}\right) \text {. }
$$

According to the above operators, we define a set of possible events as $\mathcal{E}=\left\{A_{k}, D_{k}, k=1,2,3, \ldots, K\right\}$.

Investigating $\mathrm{CAC}$ policy involves the determination of connection admission as a function of the current state $s$ and event $e \in \mathcal{E}$. This problem can be formulated as an MDP. We now give a brief description of the model.

Decision epochs take place only at arrival times. If a wavelength has sufficient free space, the connection may be accommodated immediately, or it can be rejected to preserve the capacity for the users of the other classes. Let $a \in\{0,1\}$ be the action taken after an arrival. Then, the decision maker accepts the arriving call if $a=1$. Otherwise, the call will be rejected .

Let $P_{a}, a=0,1$ be the policy operator to describe the change of system state when applying action $a$ :

$$
\text { - } A_{k} P_{a} s=\left(n_{1}, n_{2}, \ldots, n_{k}+a, \ldots, n_{K}\right) \text {. }
$$

We also define $\Pi=\left(\pi_{1}, \pi_{2}, \cdots \pi_{n}\right)$ as the call admission policy such that $\pi_{i}$ is the action applied after the $i t h$ event.

This initial continuous-time MDP can be converted into an equivalent discrete-time MDP by applying the uniformization technique [2]. To do so, we introduce a random sampling rate $\nu$ defined as [2]:

$$
\nu:=\sum_{k=1}^{K}\left(\left\lfloor\frac{T}{t_{k}}\right\rfloor \mu_{k}+\lambda_{k}\right)
$$

where $\left.\mid \frac{T}{t_{k}}\right\rfloor$ represents the maximum number of class $c_{k}$ calls that can be carried by a wavelength. After sampling the system with rate $\nu$ and considering the equivalent discretetime MDP, it can be seen that only one single transition can occur during each time slot. We define $s_{n}$ as the state of the discrete-time system during time slot $n$. A transition can correspond to an event of: 1) Class $c_{k}$ call arrival or departure event or, 2) fictitious or dummy event [2].
The next phase towards MDP formulation is to define a reward function. One can note that the blocking rates of different classes depend on their slot utilization rates. Therefore, we define the one-step reward function as:

$$
R(s)=\sum_{k=1}^{K} \alpha_{k}\left(t_{k} n_{k}\right), s \in S,
$$

where $\left(t_{k} n_{k}\right)$ represents the class $c_{k}$ slot utilization and $\alpha_{k}$ is the weighting factor assigned to this class. The choice of the weighting factor, $\alpha_{k}$, has an impact on the slot occupancy rates by different classes and their respective blocking probabilities. Note that when $\alpha_{k}=1$ for all $k=1,2, \ldots, K$, then the optimal policy aims at maximizing the total utilization of the wavelength.

Based on the one-step reward function, we can apply the results of discounted cost model with the finite horizon. In order to estimate the expected reward under policy $\Pi$ and at the time step $n$, we can define $n$-stage finite-horizon value function as:

$$
V_{n}^{\Pi}(s)=E_{s}^{\Pi}\left[\sum_{i=0}^{n-1} \gamma^{i} R\left(s_{i}\right) \mid s_{0}=s\right],
$$

where $0<\gamma<1$ is the discount factor, $\Pi=$ $\left(\pi_{1}, \pi_{2}, \cdots \pi_{n}\right)$ is the admission policy and $E_{s}^{\Pi}$ denotes the conditional expected value given that the initial state is $s$ while decision maker follows the policy $\Pi$. The optimal policy $\Pi_{o p t}(s)$ and the optimal value function $V_{n}(s)$ are given by [6]:

$$
\Pi_{o p t}(s)=\arg \max _{\Pi} V_{n}^{\Pi}(s) \text { and } V_{n}(s)=\max _{\Pi} V_{n}^{\Pi}(s) .
$$

The optimal value function can be computed by using the following recursive scheme, known as the relative value iteration algorithm [6].

$$
V_{n+1}(s)=\max _{\Pi}\left[R(s)+\gamma \sum_{s^{\prime}} P_{s s^{\prime}}^{\pi} V_{n}\left(s^{\prime}\right)\right],
$$

where $P_{s s^{\prime}}^{\pi}:=P\left(s_{n+1}=s^{\prime} \mid s_{n}=s, \pi_{n}=\pi\right)$ is the transition probability to jump from state $s$ to state $s^{\prime}$ when applying policy $\pi$. For a given random sampling rate $\nu$ and $k=1,2, \ldots, K, P_{s s^{\prime}}^{\pi}$ can be written by:

$$
P_{s s^{\prime}}^{\pi} \times \nu= \begin{cases}\lambda_{k} & \text { if } s^{\prime}=A_{k} P_{0} s, \pi=0, \\ \lambda_{k} & \text { if } s^{\prime}=A_{k} P_{1} s, \pi=1, \\ n_{k} \mu_{k} & \text { if } s^{\prime}=D_{k} s, \\ F & \text { if } s^{\prime}=s,\end{cases}
$$

where $F=\nu-\sum_{k=1}^{K}\left(n_{k} \mu_{k}+\lambda_{k}\right)$.

Replacing $P_{s s^{\prime}}^{\pi}$ in (3) yields (without loss of generality, we assume that $\nu=1$ ):

$$
\begin{aligned}
V_{n+1}(s)= & R(s)+\gamma\left[\sum_{k=1}^{K} \mu_{k} n_{k} V_{n}\left(D_{k} s\right)+F \times V_{n}(s)\right. \\
& \left.+\sum_{k=1}^{K} \lambda_{k} \max \left\{V_{n}\left(A_{k} P_{0} s\right), V_{n}\left(A_{k} P_{1} s\right)\right\}\right] .
\end{aligned}
$$


From the above equation, it can be noticed that at a class $c_{k}$ arrival time, the optimal action is $a=1$ if $V_{n}\left(A_{k} P_{1} s\right) \geq$ $V_{n}\left(A_{k} P_{0} s\right)$ and $a=0$, otherwise.

Recursively, we can determine the sequence of $n$-stage value functions $\left\{V_{1}(s), V_{2}(s), \cdots V_{n}(s)\right\}$, and the limit of this sequence when $n$ goes to infinity. Lippman in [4] shows that $V(s):=\lim _{n \rightarrow \infty} V_{n}(s)$ exists and it is the solution of the infinite horizon discounted cost problem. Besides, $V(s)$ is the unique solution to the dynamic programming (4).

\section{Structure of the optimal policy}

The Policy Iteration algorithm [6] is implemented to numerically compute the optimal policy. This section is devoted to study the effect of the weighting factor, $\alpha_{k}$, and the traffic characteristics on the optimal CAC structure.

Consider a 2-class system. Classes $c_{1}$ and $c_{2}$ include OC- 12 and OC- 48 streams, respectively. Assume that the capacity of each wavelength is OC-192. Therefore, we can divide each wavelength into 16 time slots of length OC-12 (i.e., $T=16, t_{1}=1, t_{2}=4$ ). A class $c_{2}$ call is accepted whenever at least four time slots are unused in the wavelength, whereas class $c_{1}$ calls are accommodated when only one time slot is available. For this system, the optimal value function is:

$$
\begin{aligned}
V_{n+1}(s)=R(s)+ & \gamma\left[\mu_{1} n_{1} V_{n}\left(D_{1} s\right)+\mu_{2} n_{2} V_{n}\left(D_{2} s\right)+\right. \\
& \lambda_{1} \max \left\{V_{n}\left(A_{1} P_{0} s\right), V_{n}\left(A_{1} P_{1} s\right)\right\}+ \\
& \lambda_{2} \max \left\{V_{n}\left(A_{2} P_{0} s\right), V_{n}\left(A_{2} P_{1} s\right)\right\}+ \\
& \left.\left(1-\mu_{1} n_{1}-\mu_{2} n_{2}-\lambda_{1}-\lambda_{2}\right) V_{n}(s)\right] .
\end{aligned}
$$

\subsection{Impact of weighting factors on optimal policy}

In order to investigate the effect of the weighting factors on the CAC, we fix $\alpha_{1}=1, \lambda_{1}=4 \lambda_{2}=8$ and depict the CAC policy for two values of $\alpha_{2}$. Figs. 1(a) and (b) illustrate the structure of the CAC policy at a class $c_{1}$ arrival time for $\alpha_{2}=2$ and 3, respectively. The optimal policy accepts all $c_{2}$ arrival calls in these two situations.

Note that the CAC policy has a structure of generalized switching curve. Fig. 1(a) illustrates that there exists a set of states in which the decision maker rejects $c_{1}$ calls to reserve the remaining capacity for accommodating incoming $c_{2}$ calls. We define these states, such as $(12,0),(8,1)$, $(4,2)$ and $(0,3)$, as threshold states. In threshold states, there are only four time slots available and by accepting a $c_{1}$ call in theses states, a future $c_{2}$ call is most likely to be blocked. Moreover, when the available capacity is not enough to carry a $c_{2}$ call (e.g., states $\left.(9,1),(5,2)\right)$, the decision maker tends to increase the class $c_{1}$ utilization by

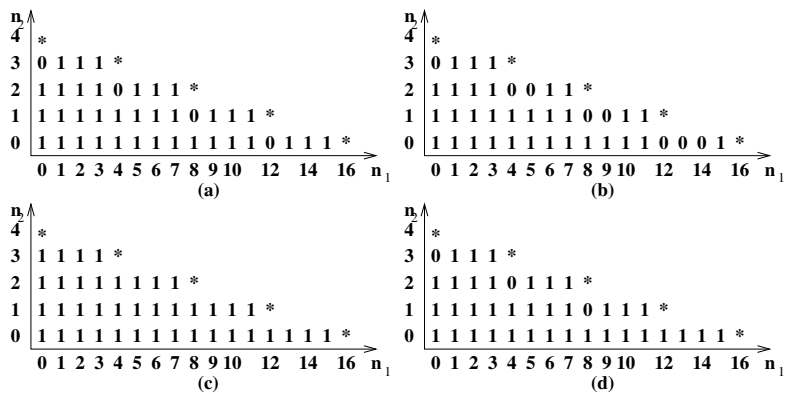

Figure 1. Representation of optimal CAC policy. Sign "*" refers to a boundary state where the system is already full and no action can be taken.

accepting $c_{1}$ calls. By increasing $\alpha_{2}$ from 2 to 3, the policy maker rejects $c_{1}$ calls not only in threshold states, but also in some other states on the neighborhood of these states. For instance, if a $c_{1}$ arrival is rejected in state $(9,1)$, then after a departure of either class, there are enough time slots to carry a $c_{2}$ call.

\subsection{Impact of arrival rates on optimal policy}

We now set $\alpha_{1}=\alpha_{2}=1, \lambda_{1}=8$ and vary $\lambda_{2}$. This case deals with maximizing the system utilization. Figs. 1(c) and (d) depict the optimal actions at an arrival epoch of a $c_{1}$ call, for $\lambda_{2}=2$ and 8 , respectively. Note that regardless of the value of $\lambda_{2}$, the CAC mechanism always accommodates an arriving $c_{2}$ call. Fig. 1(c) shows that the CAC policy accepts all $c_{1}$ calls. Therefore, the optimal policy is a CS policy for this traffic distribution. In Fig. 1(d), the CAC policy is plotted for $\lambda_{1}=\lambda_{2}$ and $t_{2}=4 t_{1}$. Note that the expected slot request rate of class $c_{2}$ is four times higher than $c_{1}$ (i.e., $\lambda_{2} t_{2}=4 \lambda_{1} t_{1}$ ). As a result, the decision maker rejects some of the class $c_{1}$ arrivals to reserve the capacity for class $c_{2}$. One can see that in threshold state $(12,0)$, the CAC decides to accept an arrival of class $c_{1}$ call while in other threshold states $(8,1),(4,2),(0,3)$, it rejects $c_{1}$ arriving calls. According to (5), we can interpret this behavior as follows. When the system's state is $(13,0)$, the rate of terminating a $c_{1}$ user from the system is $n_{1} \mu_{1}=13$ while in states $(9,1),(5,2)$ and $(1,3)$, the termination rates are 9,5 and 1 , respectively. Therefore, in state $(13,0)$, the probability of having a 4-slot free space is higher than states $(9,1),(5,2)$ and $(1,3)$ and consequently, the chance of losing a $c_{2}$ call in the future is lower. Thus, the CAC policy accepts a $c_{1}$ call in threshold state $(12,0)$.

From the above numerical results, we can conclude that the weighting factors can be set to control the class-based utilization. Therefore, the decision maker can provide differentiated services in terms of GoS or fairness. 


\section{Heuristic decomposition algorithm}

Obviously, for a large and realistic network, implementation of an MDP-based CAC mechanism is practically infeasible. Thus, we propose a four-step decomposition algorithm to extend the results of a single-link singlewavelength network to multi-link multi-wavelength tandem and ring network.

Step 1: For each hop, say hop $i$, partition the set of available wavelengths into subsets, each of which is dedicated to one of $o$ - $d$ pairs using hop $i$. Let $W_{m}$ denote the total number wavelengths in the subset dedicated to $o-d$ pair $m$. Let $M_{i}$ denote the number of the $o-d$ pairs that use hop $i$ as part of their routes, then equality $W_{1}+W_{2}+\ldots+W_{M_{i}}=W$ must be satisfied. In this paper, we consider two following examples; The first example is a 2-hop tandem network shown in Fig. 2. In this case, the set of available wavelengths is divided into two different subsets; one of which is dedicated to o-d 2, and the other one to o-d 1 and o-d 3. (Since $o-d 1$ and $o-d 3$ use separate hops, they can share the same subset of wavelengths.) More precisely, we dedicate half of the wavelengths to $o-d 2$ and the other half to the other pairs, $W_{1}=W_{2}=W_{3}=W / 2$. As the second example, we consider a 4-node unidirectional ring network depicted in Fig. 3. One can note that in this example there are one set of one-hop paths $\{o-d 1, o-d 2, o-d 3, o-d 4\}$, and two sets of non-overlapping two-hop paths $\{o-d 5, o-d$ $7\}$ and $\{o-d 6, o-d 8\}$. As a result, the total wavelengths can be partitioned into three subsets; one set is dedicated to $o$ - $d 1$ through $o-d 4$, and one set to o-d 5 and $o-d 7$, and the last set is assigned to $o-d 6$ and $o-d 8$. Therefore, we have $W_{i}=W / 3, i=1,2, \ldots, 8$.

Step 2: Assume that the total load of class $c_{k}$ for o-d pair $m$ is uniformly distributed among the $W_{m}$ wavelengths reserved to this pair. Thus, the arrival rate of class $c_{k}$ for each of the $W_{m}$ wavelengths is given by: $\lambda_{k} / W_{m}$.

Step 3: Compute the CAC policy presented in Section 3 with respect to $\lambda_{k} / W_{m}$.

Step 4: When a class $c_{k}$ call arrives to $o$ - $d$ pair $m$, using the CAC policy computed in Step 3, we determine the optimal action for each of the $W_{m}$ wavelengths, individually. If the optimal action for a wavelength is "acceptance", $a=1$, we put that wavelength in subset $\mathcal{W}_{a=1}^{m}$. If the subset $\mathcal{W}_{a=1}^{m}$ is empty, then the call will be rejected. Otherwise, one wavelength is selected randomly from subset $\mathcal{W}_{a=1}^{m}$, to carry the arrival call.

$\underset{o-d 1}{\stackrel{o-d 2}{(1})^{\text {hop } 1}(2)^{\text {hop } 2}(3)} \longrightarrow$

Figure 2. A 2-hop network topology.

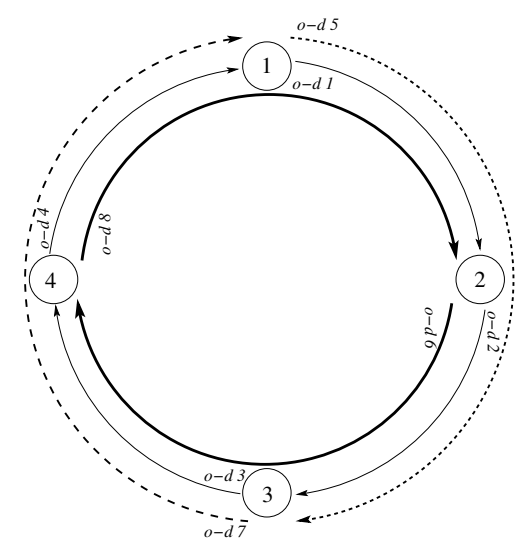

Figure 3. A 4-node ring network.

The proposed heuristic decomposition algorithm has two main advantages: 1) It masks the interferences among $o-d$ pairs by isolating them from each other. 2) It overcomes the complexity of the implementation of an MDP-based CAC mechanism, by considering each wavelength separately.

\section{Performance comparison}

In this section, the performance of the proposed heuristic algorithm without CAC implementation (i.e., using only Step 1 and Step 2 of the proposed algorithm) and with CAC implementation (i.e., using all steps of the proposed algorithm) is studied for 2-hop tandem and 4-node ring networks shown in Figs. 2 and 3, respectively. Here, we assume that the capacity of each wavelength, $T$, is 16 time slots. We will compare the performance of our proposed algorithm, with those of CS and CP policies.

We define $\rho=\sum_{k=1}^{K} \lambda_{k} / \mu_{k}$ as the offered load per $o$ $d$ pair and $B P_{k}$ as the blocking performance of class $c_{k}$ calls. Suppose that $c_{i}$ and $c_{j}$ calls experience the highest and lowest blocking probabilities in the network, then we define fairness ratio as $f_{r}:=B P_{i} / B P_{j}$.

According to Fig. 2, o-d 1 (respectively, o-d 3 ) consists of the calls that use hop 1 (respectively, hop 2), whereas $o$ $d 2$ includes the customers that use both hop 1 and hop 2 . Furthermore, the network is decomposed into 3 independent $o-d$ pairs with $W_{1}=W_{2}=W_{3}=W / 2$.

We first study the fairness of a system with two wavelengths and two classes of users, $c_{1}$ and $c_{2}$, with $t_{1}=1$ and $t_{2}=4$. One of the wavelengths is dedicated to $o-d 2$ and the other one to $o-d 1$ and $o-d 3$. We set the arrival rate of class $c_{k}$ inversely proportional to its time slot requirement. Hence, the expected slot request rates of the two classes are the same (i.e., $t_{1} \lambda_{1}=t_{2} \lambda_{2}$ ). We first simulated the system without using CAC policy. Fig. 4 depicts the class-based blocking probabilities versus offered load. One can notice that when the CAC is not used, the blocking probability of 


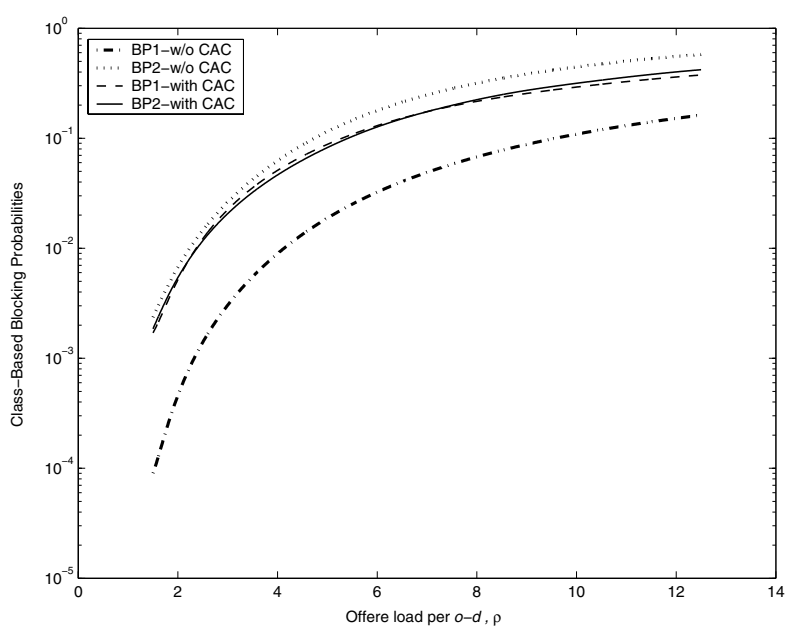

Figure 4. Class-based blocking probabilities with and without using CAC. $W=2, t_{1}=$ $1, t_{2}=4, t_{1} \lambda_{1}=t_{2} \lambda_{2}$.

class $c_{2}$ is about 10 times higher than class $c_{1}$. We will show that by applying our proposed CAC with appropriate values of $\alpha_{1}$ and $\alpha_{2}$, the fairness objective will be met. Assuming $\alpha_{1}=1$, for each offered load we find a relevant value of $\alpha_{2}$ through the simulation, so that the blocking rates of $c_{1}$ and $c_{2}$ calls become equal. For each offered load, we also obtain the structure of the optimal policy. Although the value of $\alpha_{2}$ depends on the offered load, we have noticed that the structure of the optimal policy, that results in equal blocking probabilities, remains the same as long as $\lambda_{1}=4 \lambda_{2}$ holds. For instance for $\lambda_{1}=4 \lambda_{2}=8$, we came up with the CAC policy depicted in Fig. 1(a). We used this CAC policy and simulated the system for different offered loads. Fig. 4 shows the class-based blocking probabilities versus the offered load, when implementing this CAC mechanism. It can be observed that the blocking probabilities of both classes are very close to each other. In fact, we reduce the blocking rate of $c_{2}$ calls at the expense of increasing the blocking rate of $c_{1}$ calls and a deterioration of the overall blocking probabilities in the system as illustrated in Fig. 5.

Now class $c_{3}$ is added to the network. Assuming $t_{3}=8$, we investigate the fairness ratio when $\lambda_{1}=4 \lambda_{2}=8 \lambda_{3}$. Similar to the previous case, we obtain the appropriate CAC policy in order to achieve a fairness ratio close to 1 . Table 1 (respectively, Table 2) shows the blocking probabilities (BP) of $c_{1}, c_{2}$ and $c_{3}$ calls for a system with $W=2$ (respectively, $W=10$ ). Since each $o-d$ pair is dedicated the same number of wavelengths, the blocking probability experienced by each class is the same in all $o$ - $d$ pairs. Columns $c_{1}, c_{2}$ and $c_{3}$ show the class-based blocking probabilities. Comparison of these tables illustrates that: 1) for systems without $\mathrm{CAC}$, the fairness ratio increases by increasing the

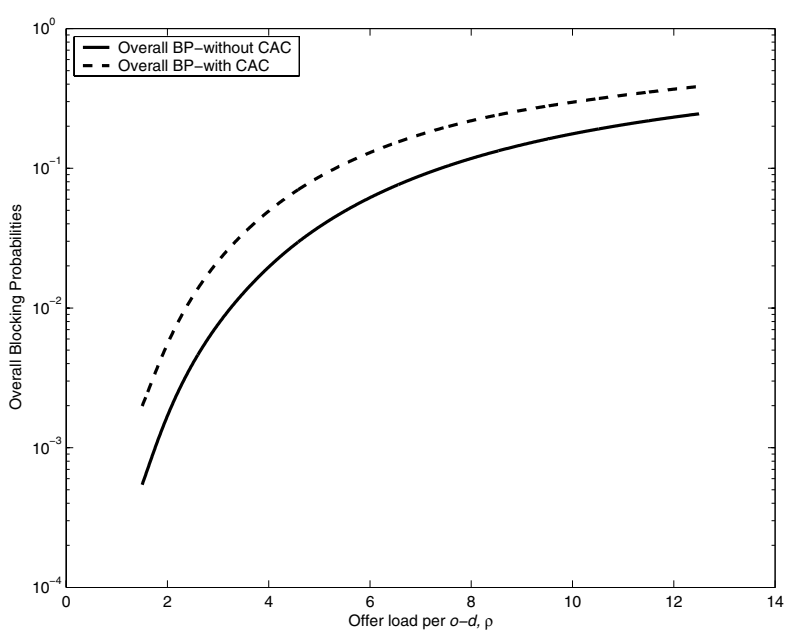

Figure 5. Overall call blocking probabilities with and without using CAC. $W=2, t_{1}=$ $1, t_{2}=4, t_{1} \lambda_{1}=t_{2} \lambda_{2}$.

number of wavelengths, 2) the difference between the fairness ratios with and without CAC implementation is larger for the 10-wavelength system in Table 2 and, 3) in all the cases, the CAC mechanism is able to maintain the fairness ratio close to 1 . One can see that $f_{r} \in[1.05,1.34]$ for $W=2$ and $f_{r} \in[1.5,1.73]$ for $W=10$.

We now compare the performance of the proposed MDPbased CAC with CP policy. This comparison is based on the number of wavelengths required to provide a certain grade of service. For a given offered load $\rho$, we apply the MDPbased CAC and determine $B P_{k}$. When applying $\mathrm{CP}$, class $c_{k}$ is allocated a fixed number of wavelengths, $w_{k}^{c p}$. The blocking probability experienced by class $c_{k}$ with traffic in-

Table 1. Blocking probabilities for a 3-class 2-wavelength 2-hop tandem network. $t_{1}=1$, $t_{2}=4, t_{3}=8, t_{1} \lambda_{1}=t_{2} \lambda_{2}=t_{3} \lambda_{3}$.

\begin{tabular}{|c|c|ccc|c|}
\hline & & BP & for all & $o-d$ pairs & \\
\cline { 3 - 5 }$\rho$ & CAC & $c_{1}$ & $c_{2}$ & $c_{3}$ & $f_{r}$ \\
\hline 2.750 & without & 0.011 & 0.076 & 0.248 & 22.5 \\
2.750 & with & 0.132 & 0.144 & 0.178 & 1.34 \\
3.347 & without & 0.018 & 0.108 & 0.322 & 17.8 \\
3.347 & with & 0.192 & 0.201 & 0.211 & 1.09 \\
4.125 & without & 0.028 & 0.153 & 0.395 & 14.1 \\
4.125 & with & 0.233 & 0.269 & 0.271 & 1.16 \\
5.500 & without & 0.053 & 0.236 & 0.522 & 9.84 \\
5.500 & with & 0.361 & 0.343 & 0.350 & 1.05 \\
6.875 & without & 0.075 & 0.311 & 0.632 & 8.42 \\
6.875 & with & 0.387 & 0.439 & 0.419 & 1.13 \\
\hline \hline
\end{tabular}


Table 2. Blocking probabilities for a 3-class 10-wavelength 2-hop tandem network. $t_{1}=1$, $t_{2}=4, t_{3}=8, t_{1} \lambda_{1}=t_{2} \lambda_{2}=t_{3} \lambda_{3}$.

\begin{tabular}{|c|c|cccc|c|}
\hline & & BP & for all & $o-d$ pairs & \multirow{2}{*}{$f_{r}$} \\
\cline { 3 - 5 }$\rho$ & CAC & $c_{1}$ & $c_{2}$ & $c_{3}$ & $f_{r}$ \\
\hline 20.62 & without & 0.000 & 0.008 & 0.098 & $>1000$ \\
20.62 & with & 0.029 & 0.031 & 0.048 & 1.65 \\
27.50 & without & .0007 & 0.039 & 0.267 & 381 \\
27.50 & with & 0.088 & 0.132 & 0.117 & 1.5 \\
34.37 & without & 0.003 & 0.104 & 0.465 & 155 \\
34.37 & with & 0.139 & 0.209 & 0.241 & 1.73 \\
\hline
\end{tabular}

tensity $\rho_{k}=\lambda_{k} / \mu_{k}$ is given by the Erlang's B formula:

$$
E\left(b, \rho_{k}\right)=\frac{\rho_{k}^{b} / b !}{\sum_{n=0}^{b} \rho_{k}^{b} / b !},
$$

where $b=w_{k}^{c p} \cdot t_{k} / T$ is the total number of class $c_{k}$ calls that can be simultaneously carried by $w_{k}^{c p}$ wavelengths. Using Erlang's B formula, we can calculate the minimum number of wavelengths so that $E\left(w_{k}^{c p} \cdot t_{k} / T, \rho_{k}\right)<B P_{k}$.

As listed in Table 2, for a 10-wavelength system with MDP-based CAC and a total offered load $\rho=27.50$, the set of class-based blocking probabilities are $B P_{1}=0.088$, $B P_{2}=0.132$ and $B P_{3}=0.117$. When applying $\mathrm{CP}$ policy, the minimum number of wavelengths required by class $c_{1}, c_{2}$ and $c_{3}$ are 2, 2 and 3 , respectively. Therefore, 7 wavelengths is required for each $o-d$ pair. As $o-d 1$ and $o-d 2$ (respectively, o-d 2 and $o-d 3$ ) use hop 1 (respectively, hop 2) as part of their routes, then we need 14 wavelengths in total, which shows $40 \%$ growth in terms of network resource cost. We also used CS policy and conducted simulations to evaluate the performance of the system. Table 3 reports the blocking performance of a 3-class 10-wavelength system with the same parameters used in Table 2 . Here, a connection with less hop counts is more likely to be accepted than one with more hop counts: One can see that class-based blocking probabilities of $o-d 1$ and $o-d 3$ are lower than the ones of $o-d 2$. Comparison of Tables 2 and 3 shows that our proposed method improves the fairness. As an example, we

Table 3. Blocking probabilities for a 3-class 10-wavelength 2-hop tandem using CS policy.

$$
t_{1}=1, t_{2}=4, t_{3}=8, t_{1} \lambda_{1}=t_{2} \lambda_{2}=t_{3} \lambda_{3} \text {. }
$$

\begin{tabular}{|l|ccc|ccc|}
\hline & BP & for & $o-d 1,3$ & BP & for & $o-d 2$ \\
\cline { 2 - 7 }$\rho$ & $c_{1}$ & $c_{2}$ & $c_{3}$ & $c_{1}$ & $c_{2}$ & $c_{3}$ \\
\hline 20.6 & .000 & 0.000 & 0.016 & .000 & 0.002 & 0.102 \\
27.5 & .000 & 0.004 & 0.104 & .000 & 0.039 & 0.410 \\
34.3 & .000 & 0.020 & 0.271 & .001 & 0.151 & 0.722 \\
\hline \hline
\end{tabular}

Table 4. Global Wavelength Utilization for a 3-class 2-wavelength 2-hop tandem network.

$t_{1}=1, t_{2}=4, t_{3}=8, \lambda_{1}=\lambda_{2}=\lambda_{3}$.

\begin{tabular}{|l|c|ccc|c|c|}
\hline & & BP & for all & pairs & & \\
\cline { 3 - 5 }$\rho$ & CAC & $c_{1}$ & $c_{2}$ & $c_{3}$ & Util. & $f_{r}$ \\
\hline 1.5 & without & 0.048 & 0.088 & 0.254 & 0.043 & 5.29 \\
1.5 & with & 0.052 & 0.092 & 0.250 & 0.044 & 5.00 \\
4.0 & without & 0.083 & 0.222 & 0.502 & 0.208 & 6.04 \\
4.0 & with & 0.302 & 0.220 & 0.450 & 0.235 & 2.04 \\
6.0 & without & 0.109 & 0.419 & 0.741 & 0.554 & 6.79 \\
6.0 & with & 0.384 & 0.546 & 0.685 & 0.603 & 1.78 \\
8.0 & without & 0.193 & 0.619 & 0.894 & 0.875 & 4.63 \\
8.0 & with & 0.575 & 0.581 & 0.861 & 0.887 & 1.49 \\
\hline \hline
\end{tabular}

compare the result of our proposed policy with CS policy when the total offered load is $\rho=27.50$. When CS policy is used, $B P_{1}$ is almost zero in all $o-d$ pairs while $B P_{3}$ for $o-d 2$ is 0.410 and for $o-d 1$ and $o-d 3$ is 0.104 . By applying the CAC, we have $B P_{1}=0.088$ and $B P_{3}=0.117$ for all $o-d$ pairs.

We now show that our proposed CAC policy can be used to improve the global utilization. We consider a system with the following parameters: $\lambda_{1}=\lambda_{2}=\lambda_{3}, t_{1}=1, t_{2}=$ $4, t_{3}=8, \alpha_{1}=\alpha_{2}=\alpha_{1}=1, W_{1}=W_{2}=W_{3}=1$ and $W=2$. Table 4 presents the utilization of the system with and without CAC. It can be observed that for all traffic loads both utilization and fairness ratio are improved. For low traffic load, the blocking rates are relatively small, in particular for $c_{1}$ and $c_{2}$, and as a result, the utilization is approximately the same with or without CAC mechanism. For high load (e.g., $\rho=8$ ), the wavelengths are almost full regardless of CAC implementation. For medium loads, $\rho=4$ and 6 , the use of MDP-based CAC results in an improvement of the utilization (e.g., up to $12.9 \%$ for $\rho=4$ ).

As the last case study, we consider the 4-node unidirectional ring network depicted in Fig. 3. Assume that $t_{1} \lambda_{1}=t_{2} \lambda_{2}=t_{3} \lambda_{3}$ and $t_{1}=1, t_{2}=4, t_{3}=8$. We first deploy the CS policy and carry out the simulation to evaluate the performance of the system. Table 5 shows the blocking rates of a 3-class 15-wavelength 4-node unidirectional

Table 5. Blocking probabilities for a 3-class 15-wavelength 4-node ring network using CS policy. $t_{1}=1, t_{2}=4, t_{3}=8, t_{1} \lambda_{1}=t_{2} \lambda_{2}=t_{3} \lambda_{3}$.

\begin{tabular}{|l|ccc|ccc|}
\hline \multirow{2}{*}{$\rho$} & BP & for & $o-d 1-4$ & BP & for & $o-d 5-8$ \\
\cline { 2 - 7 } & $c_{1}$ & $c_{2}$ & $c_{3}$ & $c_{1}$ & $c_{2}$ & $c_{3}$ \\
\hline 20 & .000 & 0.000 & 0.036 & .000 & 0.007 & 0.175 \\
25 & .000 & 0.006 & 0.135 & .000 & 0.045 & 0.429 \\
30 & .000 & 0.021 & 0.275 & .000 & 0.13 & 0.654 \\
\hline
\end{tabular}


Table 6. Blocking probabilities for a 3-class 10-wavelength 4-node ring network. $t_{1}=1$, $t_{2}=4, t_{3}=8, t_{1} \lambda_{1}=t_{2} \lambda_{2}=t_{3} \lambda_{3}$.

\begin{tabular}{|c|c|ccc|c|}
\hline & & BP & for all & $o-d$ pairs & \\
\cline { 3 - 5 }$\rho$ & CAC & $c_{1}$ & $c_{2}$ & $c_{3}$ & $f_{r}$ \\
\hline 20 & without & 0.000 & 0.007 & 0.085 & $>1000$ \\
20 & with & 0.029 & 0.028 & 0.033 & 1.17 \\
25 & without & .0003 & 0.026 & 0.198 & 660 \\
25 & with & 0.075 & 0.082 & 0.83 & 1.1 \\
30 & without & .0013 & 0.063 & 0.351 & 270 \\
30 & with & 0.126 & 0.155 & 0.162 & 1.28 \\
& & & &
\end{tabular}

ring network when CS policy is deployed. One can notice that the class-based blocking probabilities of one-hop calls (i.e., o-d 1 through $o-d 4$ ) are lower than the ones of twohop calls (i.e., o-d 5 through $o-d 8$ ). We also implemented our heuristic decomposition algorithm to improve the fairness problem for this network. Table 6 reports the result of our method. In Table 5, when CS policy is deployed and $\rho=20, B P_{1}$ is zero in all $o$ - $d$ pairs while $B P_{3}$ for $o$ - $d 1-4$ is 0.036 and for $o-d 5-8$ is 0.175 . After using our MDP-based CAC policy, we have $B P_{1}=0.029$ and $B P_{3}=0.033$ for all $o-d$ pairs which indicates the remarkable improvement in terms of fairness.

According to the above numerical results, the CAC mechanism may block one class of users to preserve the capacity for other classes. This may degrade the overall blocking performance in the system. Therefore, it is a tradeoff between improving fairness and minimizing the overall blocking probabilities.

\section{Conclusion}

We have described a call admission policy to provide fairness control and service differentiation in WDM grooming networks. The problem has been formulated as an MDP for a single-link network and the optimal policy is obtained by the Policy Iteration method. Based on properties of the optimal policy, a heuristic decomposition algorithm is proposed for multi-link networks. The heuristic algorithm has lower computational complexity with very good performance. Simulation results compared the performance of the proposed approach, with that of CS and CP policies. It has been observed that we can achieve substantial improvement in terms of fairness ratio and utilization. Although in this paper we have considered tandem and ring network topologies, our proposed decomposition algorithm can be extended to an arbitrary mesh network.

\section{References}

[1] R. A. Barry and P. A. Humblet. Models of blocking probability in all-optical networks with and without wavelength changers. IEEE J. Sel. Areas Comm., 14(5):858-867, June 1996.

[2] D. P. Bertsekas. Dynamic progrmming deterministic and stochastic models. Prentice-Hall, Englewood cliffs, 1987.

[3] A. Birman. Computing approximate blocking probabilites for a class of all-optical networks. IEEE J. Sel. Areas Comm., 14(5):852-857, June 1996.

[4] S. A. Lippman. Semi-markov decision processes with unbounded rewards. Management Science, 13(7):717-731, 1973.

[5] K. Mosharaf, J. Talim, and I. Lambadaris. A markov decision process model for dynamic wavelength allocation in wdm networks. in Proc. of IEEE GLOBECOM2003, pages 2590-2594, December 2003.

[6] M. L. Puterman. Markov decision processes. Wiley InterScience, New York, 1994.

[7] K. W. Ross and T. D. H. K. The stochastic knapsack problem. IEEE Trans. on Comm., 37(7):740-747, July 1989.

[8] R. Srinivasan and A. K. Somani. A generalized framework for analyzing time-space switched optical networks. IEEE J. of Sel. Areas Comm., pages 202-215, January 2002.

[9] V. Tamilraj and S. Subramaniam. An analytical blocking model for dual-rate sessions in multichannel optical networks. in Proc. IEEE GLOBECOM2001, pages 1515-1519, November 2001.

[10] S. Thiagarajan and A. K. Somani. A capacity correlation model for wdm networks with constrainted grooming capabilities. in Proc. IEEE ICC2001, pages 1592-1596, June 2001.

[11] S. Thiagarajan and A. K. Somani. Capacity fairness of wdm networks with grooming capabilities. Optical Networks Magazine, 2(3):24-32, May/June 2001.

[12] H. Zang, J. P. Jue, and B. Mukherjee. A review of routing and wavelength assignment approaches for wavelengthrouted optical wdm networks. Optical Networks Magazine, 1(1):47-60, January 2000.

[13] K. Zang and B. Mukherjee. A review of traffic grooming in wdm optical networks: Architectures and challenges. Optical Networks Magazine, 4(2):55-64, March/April 2003.

[14] Y. Zhu, G. N. Rouskas, and H. G. Perros. A path decomposition approach for computing blocking probabilities in wavelength-routing networks. IEEE/ACM Trans. on Net., 8(6):747-762, December 2000. 